\title{
ORBITAL ATOMIC OXYGEN EFFECTS ON MATERIALS: AN OVERVIEW OF MSFC EXPERIMENTS ON THE STS-46 EOIM-3
}

\author{
Roger C. Linton \\ Jason A. Vaughn \\ Miria M. Finckenor \\ Rachel R. Kamenetzky \\ Robert F. DeHaye \\ Ann F. Whitaker \\ Marshall Space Flight Center, AL 35812
}

\begin{abstract}
The third Evaluation of Oxygen Interaction with Materials experiment was flown on Space Shuttle Mission STS-46 (July 31 - August 8, 1992), representing a joint effort of several NASA centers, universities, and contractors.

This array of active instrumentation and material exposure sub-assemblies was integrated as a Shuttle cargo bay pallet experiment for investigating the effects of orbital atomic oxygen on candidate space materials. Marshall Space Flight Center contributed several passive exposure trays of material specimens, uniform stress and static stress material exposure fixtures, the Atomic Oxygen Resistance Monitor (AORM), and specimens of thermal coatings for the EOIM-3 variable exposure mechanisms.

As a result of 42 hours of spacecraft velocity vector-oriented exposure during the later phases of the STS-46 mission in LEO, EOIM-3 materials were exposed to an atomic oxygen fluence of $2.2 \times 10^{20}$ atoms $/ \mathrm{cm}^{2}$. In this paper, an overview is presented of the technical approaches and results from analyses of the MSFC flight specimens, fixtures, and the AORM. More detailed results from earlier EOIM missions, the LDEF, and from laboratory testing are included in associated papers of this conference session.
\end{abstract}

\section{INTRODUCTION}

The predominant natural atmospheric constituent of Low Earth Orbit (LEO) is electrically neutral atomic oxygen. Energized to a nominal $5 \mathrm{eV}$ energy by collision with orbiting spacecraft surfaces, atomic oxygen has proven to be a major factor in the degradation of a wide range of candidate spacecraft materials. Previous flight observations, including the first two EOIM flight experiments and the nearly six years of the LDEF mission, have revealed a gamut of significant material interaction effects. The flight of EOIM-3 provided an opportunity to further address $\mathrm{AO}$ and material compatibility issues in LEO for a comprehensive diversity of candidate space materials. The combination of active and passive exposure instrumentation and hardware provided means to isolate the effects of specific 
environmental factors and material interaction parameters such as mechanical stress, temperature, and the synergism of $\mathrm{AO}$ and solar UV radiation.

Post-flight characterizations of these exposed materials in comparison to pre-flight measurements have provided $\mathrm{AO}$ erosion rates and other indicators of space environmental interaction, utilizing etch profiling, mass change, reflectance and transmittance, scattering, fluorescence, and elemental and chemical composition analyses.

\section{EXPERIMENT DESCRIPTION}

Three unheated passive exposure trays containing a total of 138 material samples, including guest samples from the Jet Propulsion Laboratory, Boeing Aerospace Corporation, and Vanderbilt University were provided by MSFC. Thermal control coatings, polymeric materials, optical materials, metal foils, composites, dry film lubricants, and others were selected as candidate materials for future space missions.

Other material specimens from MSFC were included in the three EOIM-3 heated trays, which provided stable temperature control at 60,120 , and $200{ }^{\circ} \mathrm{C}$, for evaluation of thermal effects in interactions with atomic oxygen. Bulk, polished single crystal silver and copper, including both [100] and [111] crystal orientations, were exposed at all three temperatures for investigation of ordered oxidation effects. Other materials flown included aluminum anodizations, aluminum/lithium alloys, dry film lubricants, metals, thermal control coatings, and selected polymeric materials, including candidate Space Station seal materials. Considerations of intrinsic thermal decomposition and tray space allotments restricted the exposure of many of these materials to only one or two of the set temperature trays.

An approach to actively monitoring atomic oxygen flux stability was provided by the Atomic Oxygen Resistance Monitor (AORM). This device operated by measuring the electrical conductance of exposed carbon sensors (Fig. 2). As the carbon sensors were eroded by atomic oxygen, the decrease in conductance was proportional to the integrated fluence, and the rate of change was proportional to flux levels. The AORM experiment had six sensor units, each with an exposed carbon element. All units incorporated a protected carbon resistor element to provide temperature compensation. Three sensor units were heated to a constant temperature, in addition to having a compensating resistor. This was done to determine if constant temperature operation and resistance compensation were both required to produce unambiguous results from the AO sensors. During the EOIM-3 mission, all six of the AORM sensor units operated successfully throughout the 42 hours of atomic oxygen exposure, indicating the viability of both the heated and unheated units for use as a practical $\mathrm{AO}$ monitoring system.

MSFC provided Chemglaze A-276 and Z-306 coating samples for the EOIM-3 Solar Ultraviolet (SUV) Tray and the Variable Exposure Tray (VET) assemblies. These samples were covered by $90 \%$ transmissive nickel wire mesh to provide a grid pattern of unexposed material following atomic oxygen exposure for erosion etch profiling. Controlled shuttering of the SUV experiment provided continuous exposure for one set of samples and alternate exposures for selected material samples during solar illumination and shadowed phases of the EOIM-3 active mission to investigate the effects of solar UV radiation and atomic oxygen, individually and in combination. The VET experiment was similarly designed with shutters 
to sequentially expose material samples to atomic oxygen for increasing intervals to evaluate induced surface recession rates as a function of cumulative $\mathrm{AO}$ exposure. A failed microswitch in this experiment resulted in all samples receiving full exposure during the mission.

Completing the MSFC experiment elements were two types of fixtures for investigating enhanced reactivity in stressed polymeric materials exposed to atomic oxygen. Two uniform stress fixtures were designed to maintain a constant pre-loaded mechanical stress on sheets of Kapton HN by means of a spring-loaded mechanism. Two static stress fixtures provided only an initial pre-loaded strain on Kapton $\mathrm{HN}$ sheets. The erosion induced by atomic oxygen across the sheet materials has been determined by mass loss measurements for comparison to unstressed material reactivity.

\section{ENVIRONMENTAL EXPOSURE}

Space environmental exposure of the pallet experiment EOIM-3 featured 42.25 hours of flight operations while the Space Shuttle cargo bay was oriented within 2 degrees of the RAM velocity vector at an altitude of 123-124 nautical miles. The cumulative atomic oxygen fluence for the entire mission, $2.2 \times 10^{20}$ atoms $/ \mathrm{cm}^{2}$, includes a minute additional contribution (approx. 0.5\%) during deployment and associated operations of the European Retrievable Carrier (EURECA).

A total of only 30.6 equivalent sun hours (ESH) of solar electromagnetic radiation for the entire mission (7.6 ESH during RAM exposure) was computed for the payload', although this level proved sufficient to stimulate perceptible effects in a number of UV-sensitive materials. During the eight days of Shuttle cargo bay thermal vacuum exposure, passive tray temperatures varied from -5 to $+80^{\circ} \mathrm{C}$ (ibid). EOIM-3 materials were subjected to presumably benign levels of ionizing radiation, and no significant impact features resulting from meteoroid or space debris have been identified on MSFC elements of the experiment.

\section{CONTAMINATION OBSERVATIONS}

Visible evidence of molecular contamination on MSFC samples and hardware resulting from exposure during the EOIM-3 mission indicated localized effects without major widespread deposition. A faint, colorless streak pattern, more clearly distinguished under black light illumination (Fig. 3), was observed on the Kapton sheet material of one of the static stress fixtures. Analysis of solvent wipes from the stress fixtures themselves revealed the presence of silicone on the static stress fixture with the contaminated Kapton, without detection of contamination from the other fixtures. Very small droplet pattern spots, in some cases revealed as liquid splash sites under visual magnification, were found on a few, selected samples, including some of the optical samples (Fig. 4).

Each of the MSFC passive trays and the two lower temperature heated trays on EOIM-3 contained one or more magnesium fluoride overcoated aluminum mirrors as optical witness samples. The inclusion of these witness samples among the optical, thermal control, and polymeric material sample trays were intended to provide a basis of comparison for localized 
contamination effects from the different classes of materials. Diffuse reflectance measurements at wavelengths transmitted through air $(0.25-2.5 \mu \mathrm{m})$ provided no significant evidence of degradation for the witness mirrors on either passive or actively heated trays.

Potentially more sensitive measurements of the optical properties at vacuum UV wavelengths $(121.6-200 \mathrm{~nm})$ indicated patterns of increased reflectance over certain wavelengths for passive tray $\mathrm{MgF}_{2} / \mathrm{Al}$ mirrors (Fig. 5) and decreased reflectance over certain wavelengths for those from the heated trays. No significant differences in reflectance were measured between exposed and unexposed (covered) areas of the half-exposed $\mathrm{MgF}_{2} / \mathrm{Al}$ mirrors, within experimental error. Elemental and chemical composition analyses of these and other types of mirrors, including Surface Analysis by Laser Ionization (SALI), ESCA, and Rutherford Backscattering (RBS), indicate molecular contamination common in level to the small levels of "adventitious" hydrocarbons found on all samples exposed to the atmosphere of conventional laboratories.

Contamination in the form of particulate and fibrous debris was found on all of the flight optical samples. The number and distribution of particle sizes ranged from Level 500 to Level 700, MIL-SPEC-1246, in exposed sample areas compared to an average Level 500 for unexposed sample areas. These different levels of debris were detectable with bi-directional reflectance distribution function (BRDF) measurements but did not significantly degrade the specularity of most of the affected optics.

\section{POLYMERS}

A select assortment of thin film and bulk polymeric materials were included in both the passive and actively heated trays of EOIM-3. Determinations of the atomic oxygen reaction efficiency of these materials were made on the basis of both weight loss and erosion etch depth. Individual samples were placed in vacuum, then weighed immediately after removal from vacuum. Mass change calculations were based on time dependence of weight measurements with extrapolation to zero for the "true" weight. The calculated reaction efficiencies of polymers based on weight loss are, for these samples, considered more accurate than the etch depth profiles that were compromised by stylus drag through the softer materials; the inherently stiffer Lexan polycarbonate is the major exception. These results are tabulated and discussed in comparison to results from previous EOIM flights, the LDEF, and laboratory atomic oxygen testing in the associated paper of this session reviewing polymeric materials.

Preliminary results indicate no definitive difference in erosion for the Kapton sheet material from the uniform stress fixtures compared to similar material from the passive tray exposure. Kapton erosion from the relatively lightly loaded static stress fixtures was slightly higher than passively exposed material. The extent of the role of additional atomic oxygen incident scattered from the underside of the fixtures is being investigated.

Bulk material samples of candidate Space Station seal materials, silicone S383 and Viton 747 , were exposed on the $60^{\circ} \mathrm{C}$ tray only. Laboratory testing of these types of materials at $\mathrm{MSFC}^{3}$ have included the effects of thermal vacuum, AO, UV, and combinations of these exposures, based on changes in seal material permeability, Shore A hardness, fluorescence, and mass. The effects of flight exposure of these materials are discussed in relation to these tests in the associated paper of this session reviewing results for polymers. 
AO exposure-induced changes in the resistance of black, carbon-impregnated Kapton have proven to be a relatively reliable and accurate alternative gauge of atomic oxygen fluence in asher and beam facility testing at MSFC. The electrical properties change in inverse proportion to the loss by erosion of the carbon. The utility of this approach for LEO exposure was demonstrated by passive exposure on STS- 41 as part of the ISAC experiment ${ }^{4}$. The flight of EOIM-3 provided the opportunity not only to expose and evaluate multiple specimens of black Kapton, but also to evaluate the function of the active AORM, based on similar principles.

\section{THERMAL CONTROL MATERIALS}

Thermal control coatings included in the complement of MSFC samples included several new formulations of polyurethane-based paints provided by Lord Chemical Corp., two formulations of the ceramic-based radiator coatings Z-93 and YB-71, a variety of aluminum anodizations prepared by different processes, and beta cloth.

Erosion of Chemglaze A-276 and Z-306 coatings, exposed through $90 \%$ transmissive nickel wire mesh on the EOIM-3 variable exposure mechanisms (VET/SUV), was comparable in magnitude to predictions based on previous EOIM and LDEF data. No significant differences in erosion were found (as expected) for VET exposure samples, since the shutters failed to activate properly. Weight loss measurements of SUV samples indicate a systematic trend with shuttered exposure for the samples exposed continuously, samples exposed only to $\mathrm{AO}$, and samples exposed to AO only during the few hours when solar UV was detected. See Table 1.

Anodized 6061-T6 aluminum coatings were included in the passive trays and each of the actively heated trays. These coatings included representative samples prepared by three basic anodization processes using chromic, sulfuric, and boric/sulfuric acid, including ten minute and twelve minute processing times for the boric/sulfuric anodized samples. The measured changes in solar absorptance and infrared thermal emittance are reviewed in the associated paper for thermal control coatings ${ }^{11}$.

Beta cloth has an extensive history of effective utilization in space, including the cargo bay of the Space Shuttle and the LDEF ${ }^{6}$. Several types of beta cloth were flown, including material with heat-rejecting thin film aluminum backing. Thermo-optical properties were basically unchanged, as expected, for this level of exposure; the predicted loss of the TFE Teflon matrix due to the EOIM-3 level of atomic oxygen is approximately one tenth of a micron.

One of the more sensitive indicators of induced environmental interaction for many thermal control coatings is fluorescence. For these materials, exposure to the space environment, including thermal vacuum, atomic oxygen, and solar UV radiation, results in changes in the spectral emission of fluorescent radiation. The flight of EOIM-3 provided an opportunity to verify the nature and degree of effects found in samples from the LDEF, from laboratory testing, and long-stored samples from earlier Shuttle flights dating to STS-5 (1982).

For each thermal coating material investigated, the fluorescence effects of EOIM-3 exposure, with a moderate fluence of atomic oxygen and a relatively minimal exposure to solar UV radiation, are identical in nature, differing mainly in degree of effect, to altered 
fluorescence effects of similar materials exposed on $\mathrm{LDEF}^{7}$. In the laboratory, fluorescence in polyurethane-based coatings is significantly altered by a nominal exposure, in vacuum, to simulated solar UV radiation within no more than an hour of irradiation.

Common bands of fluorescent emission and similarities in response to exposure, suggesting degrees of material commonality, were found in the polyurethane paints submitted by Lord Chemical Corp., including the Chemglaze and Aeroglaze coatings, with few exceptions. Material dependent differences in fluorescence, including resolution of structure, comparative emission intensities, and additional emission band structure are found for each of the paint types. Relatively minor changes in the fluorescence of zinc orthotitanate indicate little, if any, effect on this material for the EOIM-3 level of exposure.

\section{OPTICS}

A variety of thin film and bulk optical materials were included in both the passive and actively heated trays of EOIM-3. Effects of exposure are being investigated for optical materials ranging from thin metal foil extreme UV (EUV) filters to $\mathrm{NaCl}$ and $\mathrm{KBr}$ infrared windows. A majority of the samples were thin film and bulk material optics for vacuum UV applications and a series of candidate thin film mirror coatings for X-ray applications. Characterization techniques included spectral reflectance and transmittance, fluorescence, BRDF, ellipsometry, microscopy, and chemical composition analysis. A variety of instrumented facilities were utilized to measure spectral, specular and/or diffuse reflectance and transmittance from vacuum UV through near-IR wavelengths $(110-2500 \mathrm{~nm})$. Chemical analyses included FTIR, X-ray fluorescence, ESCA, Auger, RBS, and SALI.

The candidate thin film reflectors for X-ray application included platinum, gold, iridium, and nickel films on optically flat substrates of the glass ceramic Zerodur, the selected substrate material for the NASA AXAF-I mirror assembly. While the x-ray reflecting layers for AXAF-I (currently iridium) are intended for extreme grazing incidence application with a thickness of only $25 \mathrm{~nm}$, opaque mirrors were included with the EOIM-3 samples to simplify optically intrusive analyses such as ellipsometry.

Some of the four types of candidate $\mathrm{X}$-ray mirror materials indicate some degradation in diffuse reflectance $(0.25-2.5 \mu \mathrm{m})$. Iridium and gold mirrors exposed on the passive trays were not measurably changed at these wavelengths. Small changes in reflectance of the nickel mirrors at these wavelengths indicated some effects of exposure. The more significant and temperature-proportional degradation of the gold mirrors from the 120 and $200{ }^{\circ} \mathrm{C}$ heated trays provided clear evidence of a thermally dependent degradation. At vacuum UV wavelengths, the reflecting properties of the platinum mirrors were not measurably changed. One of the iridium mirrors was unaffected, while another was significantly degraded for wavelengths less than $180 \mathrm{~nm}$. Analysis of the composition of this iridium mirror, using Auger and XPS techniques ${ }^{8}$, provided evidence for the presence of iridium oxide, $\mathrm{IrO}_{2}$ on the mirror surface. The gold mirrors flown on the heated trays are more affected at vacuum UV wavelengths, in agreement with overlapping diffuse reflectance measurements. The optical measurements and chemical composition analyses ${ }^{10}$ indicate that the dominant mechanism affecting these mirrors is diffusion or migration of the underlying nickel to the surface of the gold layer, possibly aided in transport by atomic oxygen. There is evidence of very short wavelength degradation in the gold mirrors flown on the passive trays. 
Significant changes at the vacuum UV wavelengths were also found for the nickel mirrors. Results of combined optical and chemical analyses indicate that the nickel mirrors were severely degraded by natural oxidation in the atmosphere and that additional nickel oxide was grown as a result of atomic oxygen exposure. The effects of exposure of these samples are discussed in detail in the associated optics paper in this conference session (ibid).

A luminescing phosphor of widespread utility for vacuum UV applications, sodium salicylate was found to be thinner post-flight. Visual contrast was striking under black light illumination for the exposed and unexposed areas of the partially exposed sample. Spectrofluorometer measurements of the glow stimulated by $260 \mathrm{~nm}$ incident light indicated only a slight change in fluorescence. Measurements of the fluorescence stimulated by vacuum UV wavelengths $(120-200 \mathrm{~nm})$ indicated as much as $50 \%$ loss in detected emission.

\section{SUMMARY AND CONCLUSIONS}

All of the MSFC elements of EOIM-3 functioned successfully and have provided material interaction results for a comprehensive range of candidate spacecraft materials. The six carbon sensors of the AORM functioned as designed with decreasing conductance proportional to the time integrated atomic oxygen flux (fluence) throughout the active phases of the EOIM-3 mission. Effects of mechanical stress on Kapton $\mathrm{HN}$ reactivity to atomic oxygen have been found to be negligible for the uniform stress fixtures, while slightly increased reactivity was found for Kapton $\mathrm{HN}$ in the static stress fixtures. The relative contribution of back-surface reflected atomic oxygen to the induced mass loss of Kapton HN from these fixtures is still being investigated.

Uncoated polymeric materials reacted in varying degrees of agreement with previous flight data and results from ground-based atomic oxygen testing. Reaction efficiencies for some of the polymers investigated, including Kapton and Teflon (TFE and FEP), are slightly higher than previously reported from previous EOIM flight experiments. Results indicate that coatings of hexamethyl disiloxane (HMDS) and indium tin oxide (ITO) protected the underlying Kapton from attack.

Candidate Space Station seal materials of Viton V747 and Silicone S383 were only slightly affected by the space exposure, as gauged by the small changes in Shore A hardness, helium permeability, and the thermophysical properties of solar absorptance and thermal emittance.

Thermal control ceramic-based coatings proved to be highly resistant to the EOIM-3 levels of space environment exposure. Thermo-optical properties were relatively stable for all but two of the newer formulations of the polyurethane-based coatings. No significant changes in the appearance or optical properties of the various types of anodized aluminum were detected except for the darkening of the organic dyed samples and a single chromic acid anodized aluminum sample from the $120^{\circ} \mathrm{C}$ tray.

The appearance, solar absorptance, and infrared thermal emittance of beta cloth samples were basically unchanged as a result of EOIM-3 exposure.

The diversity of analytical characterization techniques proved useful in the detection and identification of environmentally induced effects for the metallic and optical samples. Mass loss/gain measurements are revealing for some samples, such as the comparative losses for aluminum/lithium 2090 alloy samples from the heated trays compared to the similar Weldalite 
alloy samples from the passive trays, possibly indicating an effect of space exposure. Optical measurements do not indicate significant changes for these samples, while, for example, the changes in solar absorptance and/or thermal emittance of the silver and copper samples are obvious indicators of the expected oxidation. The thermally driven diffusion or migration of adhesion layer nickel into thin film gold samples was indicated by optical measurements but not confirmed until SALI profiles and RBS analysis showed nickel and nickel oxide on the surface and in the bulk of the gold layer. As a final example, the longer wavelength diffuse reflectance measurements of thin film nickel proved relatively insensitive to the oxidation revealed in much shorter wavelength (VUV) measurements and SALI investigations.

The exposed vacuum UV phosphor, sodium salicylate was found to be noticeably thinner, and the measured fluorescence stimulated by vacuum UV wavelengths decreased approximately $50 \%$ in the exposed area.

Molecular contamination, as indicated by ESCA sputtering depth profiles, was detected as an organic silicone approximately $7 \mathrm{~nm}$. thick on selected witness samples.

\section{ACKNOWLEDGMENTS}

The candidate X-ray reflector coatings were prepared by Dr. Alan Shapiro (MSFC). Special appreciation is expressed to Ed White (MSFC) for design and fabrication support, including the stress fixtures. Others who provided substantial technical contributions include Dr. Michael Bojack (Auburn University) for Auger analysis, Dr. John Williams (Auburn University) and David Edwards (MSFC) for RBS analysis of gold/nickel samples, Dr. Chris Becker (SRI) for SALI analysis, Dr. Kijoon Chang (Alabama A\&M) for providing the ellipsometer, and Perry Gray (Microcraft) for helium permeability testing and other technical support. Others from MSFC who contributed to the success of this experiment include Ralph Carruth, James Coston, Diep Trinh, Tenina Bili, Whitney Hubbs, Don Burch, and Dewitt Burns. The authors gratefully acknowledge the efforts of the JSC and KSC individuals for experiment integration and interface, with particular mention of Dr. Steven Koontz, James Visentine, and Steven Dansby of JSC.

Table 1. Space Environment Effects on SUV A-276 Paint Samples

\begin{tabular}{|c|c|c|c|}
\hline Sample & Environment & $\Delta$ Mass (mg) & DThickness $(\AA)$ \\
\hline C-SUV & Entire mission & 0.69 & 14,100 \\
\hline L-SUV & $\mathrm{AO}$ only in presence of $\mathrm{UV}$ & 0.60 & 11,900 \\
\hline D-SUV & AO only, no UV & 0.33 & 6,600 \\
\hline
\end{tabular}




\section{REFERENCES}

1. Leger, L.J., "Evaluation of Oxygen Interaction with Materials III (EOIM-III) Flight Experiment Update," JSC memo ES5-93-118, July 2, 1993.

2. Leger, L.J., Koontz, S.L., Visentine, J., Hunton, D., "An Overview of the Evaluation of Oxygen Interaction with Materials-Third Phase (EOIM-III) Experiment: Space Shuttle Mission 46," AIAA-93-0497, January 1993.

3. Linton, R.C., Finckenor, M.M., Kamenetzky, R.R., Gray, P., "Effects of Atomic Oxygen and Ultraviolet Radiation on Candidate Elastomeric Materials for Long-Duration Missions - Test Series No. 1", NASA TM-108408, June 1993.

4. Koontz, S., King, G., Dunnet, A., Kirkendahl, T., Linton, R., Vaughn, J., "The International Telecommunications Satellite (INTELSAT) Solar Array Coupon (ISAC) Atomic Oxygen Flight Experiment: Techniques, Results, and Summary," Centre National D'Etudes Spatiales, Toulouse, France, 1992.

5. Visentine, J., comp.,"Atomic Oxygen Effects Measurements for Shuttle Missions STS-8 and STS-41G, Vol. III", NASA TM-100459, Sept. 1988.

6. Linton, R.C., Whitaker, A.F., Finckenor, M.M., "Space Environment Durability of Beta Cloth in LDEF Thermal Blankets," LDEF Materials Results for Spacecraft Applications Conference, Oct. 1992.

7. Linton, R.C., Whitaker, A.F., Kamenetzky, R.R. "Fluorescence Observations of LDEF Exposed Materials as an Indicator of Induced Material Reactions," LDEF Materials Results for Spacecraft Applications Conference, Oct. 1992.

8. Williams, J.R., Bozack, M.J., Fromhold, A.T., Technical Progress Report for NASA Contract NAS8-39131, Delivery Order \#3, Auburn University.

9. Kamenetzky, R.R., Linton, R.C., Finckenor, M.M., Vaughn J.A., Whitaker, A.F., "Effects of Atomic Oxygen on Polymeric Materials Flown on EOIM-3", AIAA 93-4103.

10. Vaughn, J.A., Linton, R.C., Finckenor, M.M., Kamenetzky, R.R., "Evaluation of Atomic Oxygen Effects on Metals and Optical Thin Films on EOIM-3", AIAA 93-4104.

11. Finckenor, M.M., Linton, R.C., Kamenetzky, R.R., Vaughn, J.A., "Thermal Control Materials on EOIM-3", AIAA 93-4101.

12. Franzen, W., Brodkin, J.S., Sengupta, L.C., Sagalyn, P.L., "Ellipsometric Study of Oxide Films Formed on LDEF Metal Samples," NASA CP-3134, June 1991. 


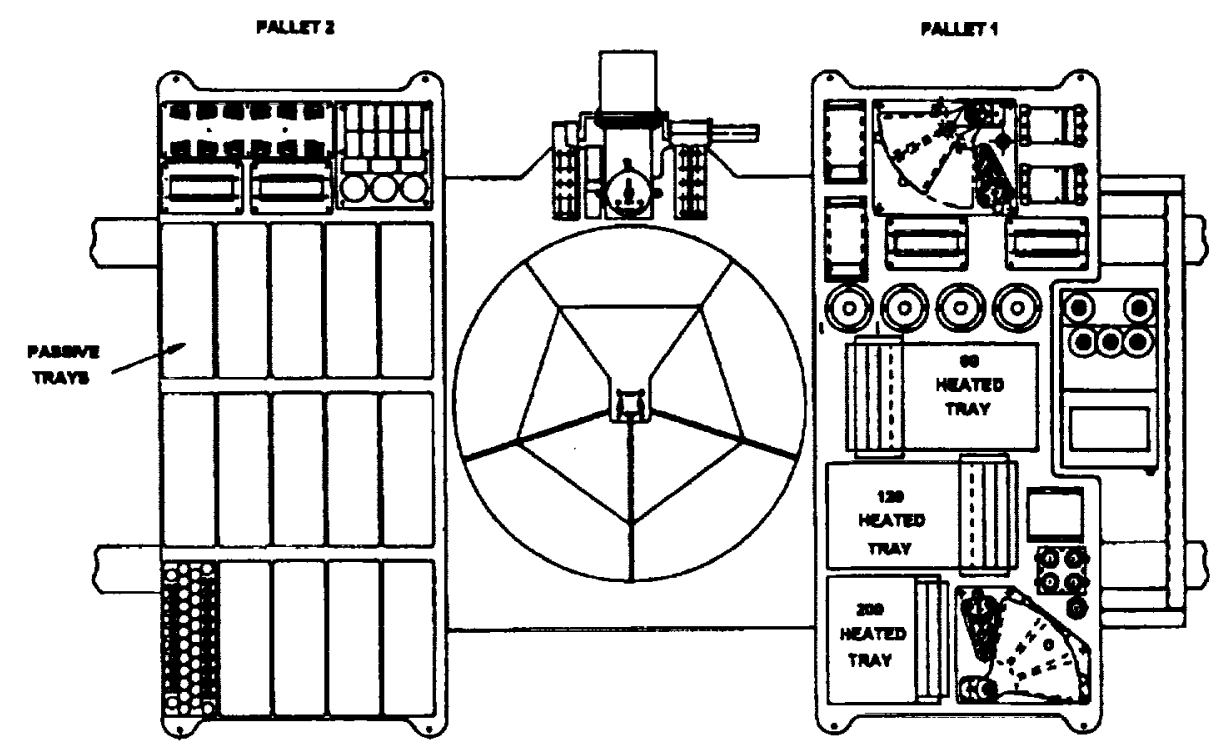

Figure 1 EOIM-3 Payload Configuration

AORM SENSOR DATA

VOLTAGE OUTPUT VS. ORBITAL EXPOSURE TIME

OUTPUT VOLTS

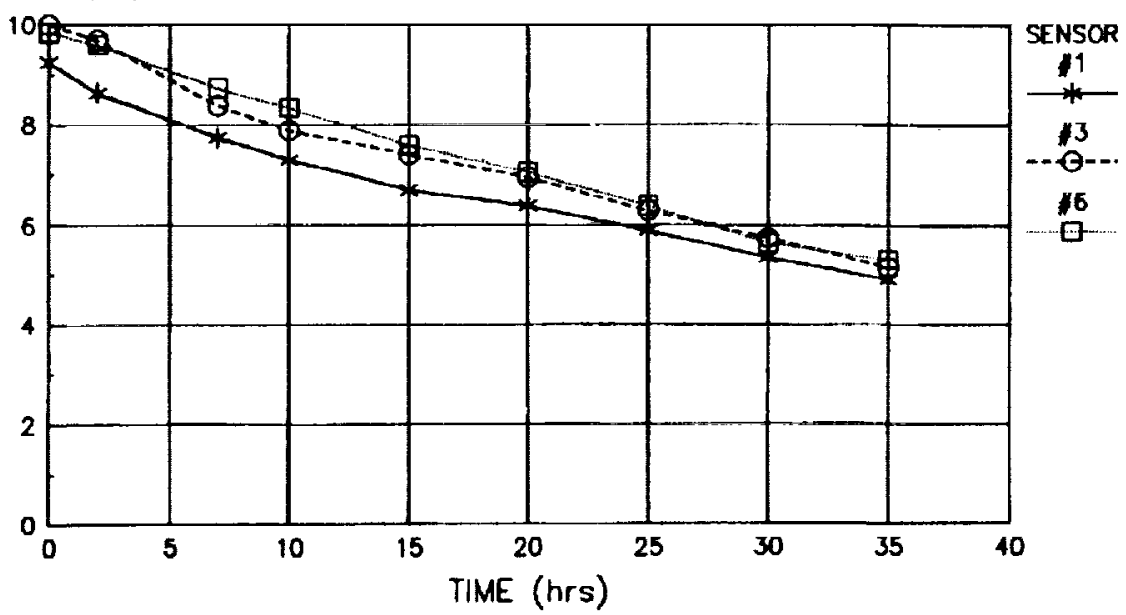

8-6-92

Time ofter EOIM-3 ON

Figure 2 Effective Conductance Decrease with AO Exposure 


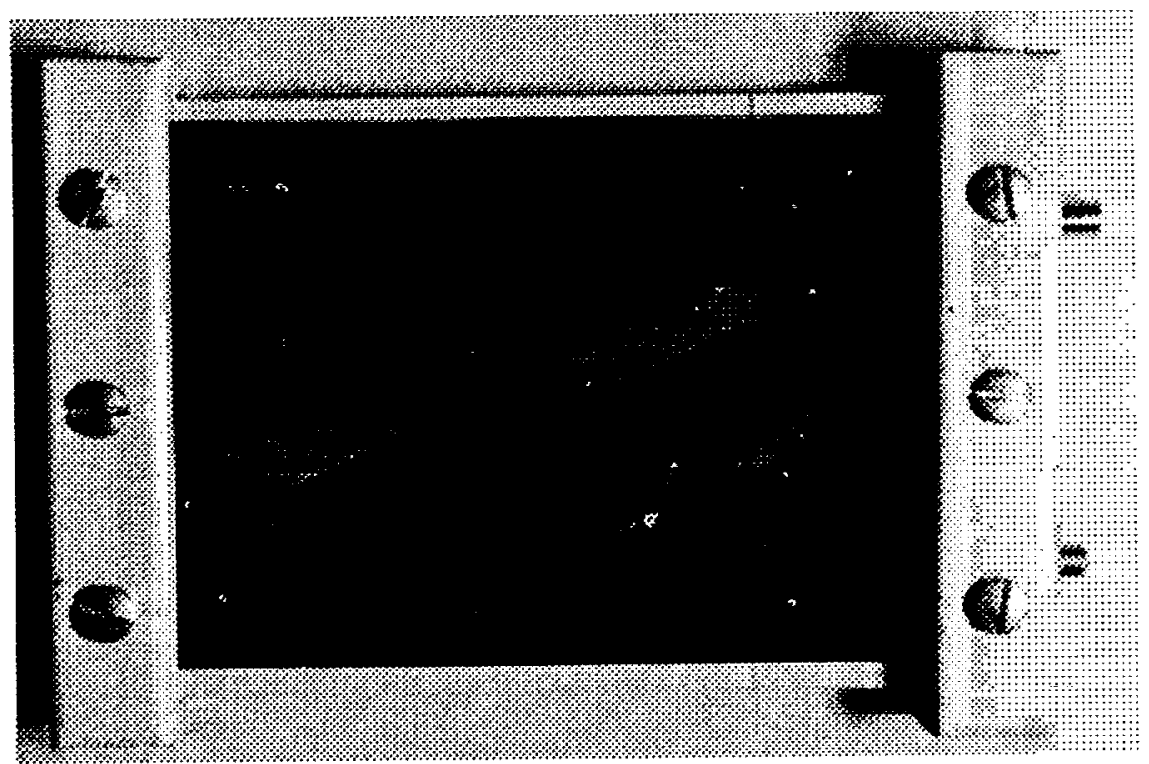

Figure 3

Contaminated Static Stress Fixture Under Black Light Illumination

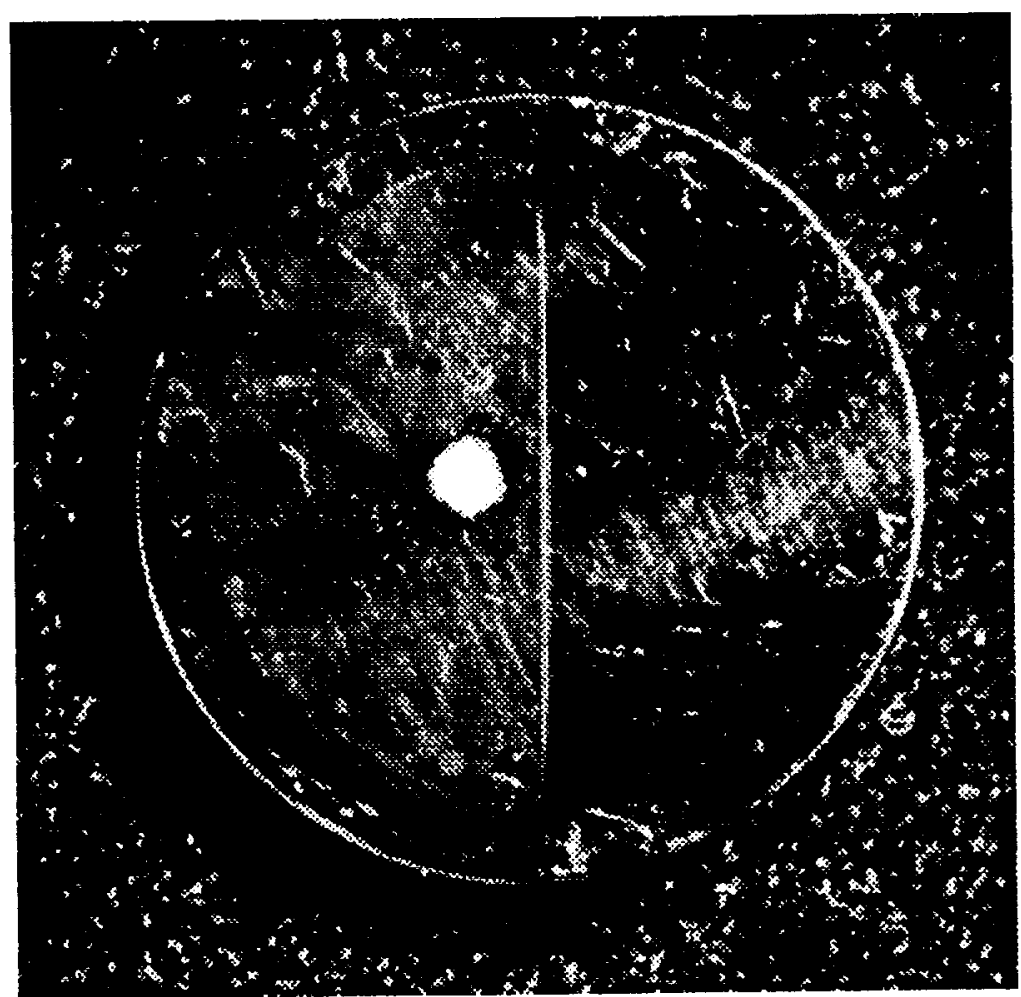

Figure 4

Contamination on Gold Mirror 


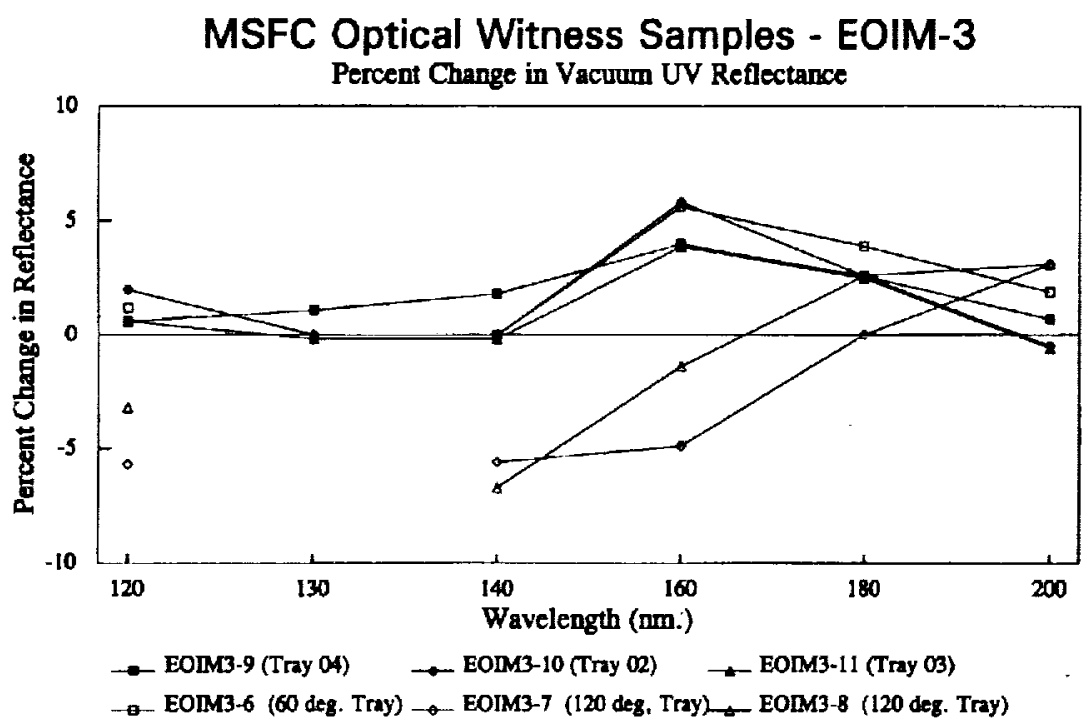

Figure 5

VUV Reflectance of $\mathrm{MgF}_{2} / \mathrm{Al} /$ Fused Silica Mirrors Optical Witness Samples on EOIM-3

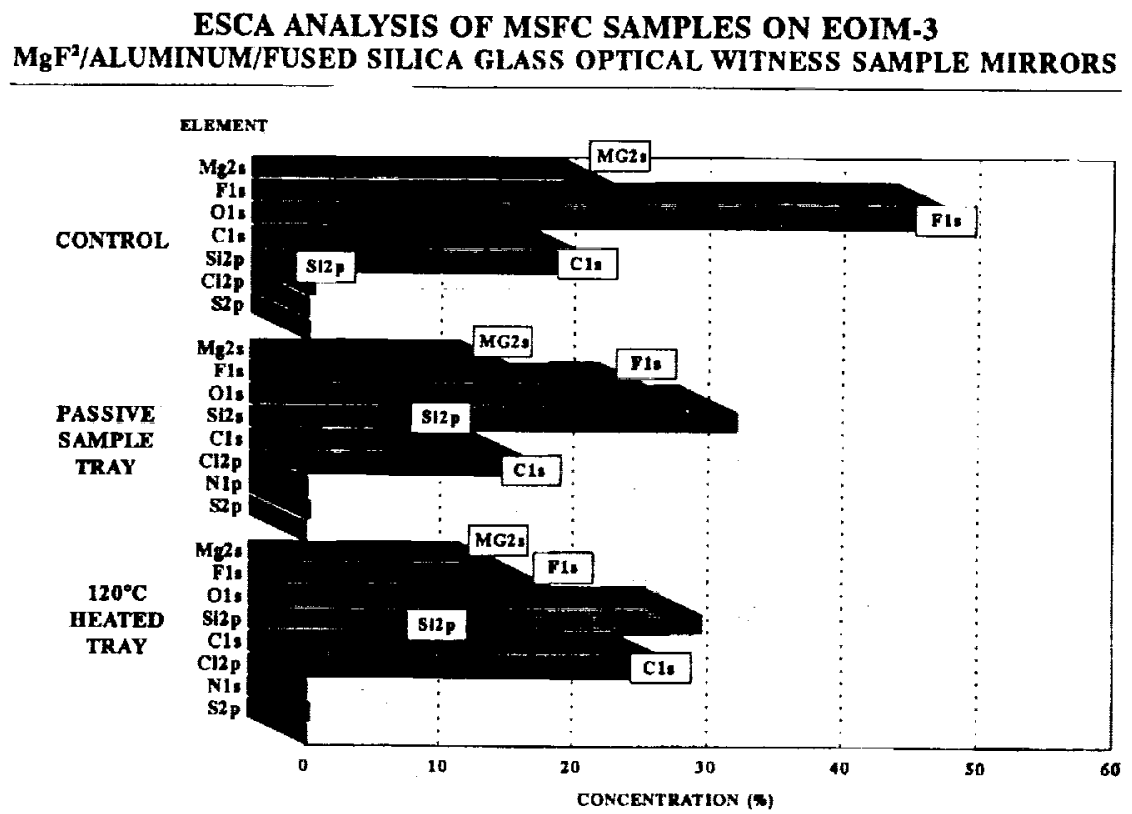

Figure 6

ESCA Analysis Results

EOIM-3 Optical Witness Samples 